\title{
Characterization of Antinociception Produced by Glutamate Microinjection in the Nucleus Tractus Solitarius and the Nucleus Reticularis Ventralis
}

\author{
A. Randich, ${ }^{1}$ M. G. Roose, ${ }^{1}$ and G. F. Gebhart ${ }^{2}$ \\ Departments of ${ }^{1}$ Psychology and PPharmacology, The University of lowa, lowa City, lowa 52242
}

Four experiments examined the role of the nucleus tractus solitarius (NTS) and subjacent nucleus reticularis ventralis (NRV) in the production of antinociception. Experiment 1 showed that microinjection of glutamate $(50 \mathrm{nmol})$ into the caudal NTS resulted in inhibition of the tail-flick reflex, hypotension, and mild bradycardia, whereas microinjection of glutamate into the rostral NTS resulted only in hypotension and mild bradycardia. Microinjections of glutamate into the NRV resulted in inhibition of the tail-flick reflex, hypertension, and mild bradycardia. Experiment 2 demonstrated that the magnitude of the antinociceptive and cardiovascular responses resulting from glutamate microinjections into both the depressor and pressor regions were dose dependent. Experiment 3 showed that the antinociceptive effects resulting from microinjections of glutamate into either the depressor or pressor regions could not be antagonized by phentolamine $(30 \mu \mathrm{g})$, methysergide (30 $\mu \mathrm{g})$, or naloxone (30 $\mu \mathrm{g})$ alone, but the combined intrathecal administration of phentolamine and methysergide $(7.5,15$, or $30 \mu \mathrm{g}$ of each) attenuated the antinociception resulting from microinjection of glutamate into either depressor or pressor regions in a dose-dependent fashion. Experiment 4 showed that systemic administration of hexamethonium blocked the pressor response produced by microinjection of glutamate into the NRV but did not reduce the antinociceptive effect of the microinjection. These findings are consistent with a role for the NTS and NRV in the production of antinociception.

Several lines of evidence support the view that the nucleus tractus solitarius (NTS) serves as a medullary substrate involved in the production of antinociception. First, electrical stimulation in the NTS results in inhibition of the tail-flick reflex evoked by noxious radiant heat, and this inhibitory effect can be antagonized by either systemic or intrathecal administration of naloxone (Lewis et al., 1987; Lohof et al., 1987). In a preliminary study from our laboratory, microinjection of glutamate into the NTS of the rat also resulted in inhibition of the tail-flick reflex, hypotension, and mild bradycardia (Randich and Aicher, 1988). Finally, electrical stimulation in the NTS inhibits responses of spinal dorsal horn and lamina $X$ neurons to electrical stimulation of hindlimb nerves in cat, and this inhibitory effect can

Received Dec. 3, 1987; revised Apr. 6, 1988; accepted Apr. 13, 1988.

This research was supported by NIH Grant NS 22966 to A. R. We gratefully acknowledge the assistance of the Psychology Department Shop.

Correspondence should be addressed to Dr. Alan Randich at the above address. Copyright (C) 1988 Society for Neuroscience $0270-6474 / 88 / 124675-10 \$ 02.00 / 0$ also be antagonized by systemic administration of naloxone ( $\mathrm{Du}$ and Zhou, 1987).

Second, vagal afferents terminate in the region of the NTS (Kalia and Sullivan, 1982), and numerous studies have demonstrated that electrical, pharmacological, and physiological activation of vagal afferents results in hypotension, bradycardia, and antinociception as indexed by both bchavioral and electrophysiological measures (Ammons et al., 1983a, b; Maixner and Randich, 1984; Randich and Maixner, 1984a, b; Ren et al., 1988). For example, inhibition of the tail-flick reflex in the rat produced by electrical stimulation of vagal afferents is (1) dependent upon the frequency, intensity, and duration of stimulation; (2) blocked by local anesthetic microinjections into either the NTS, the nucleus raphe magnus (NRM) - medullary reticular formation (MRF), the nucleus raphe obscurus (NRO), or the lateral reticular nuclei (LRN); (3) blocked by administration of the nonspecific glutamate receptor antagonist $\gamma$ - $d$-glutamylglycine (DGG) into the NTS; and (4) blocked by the intrathecal administration of either naloxone or the combination of phentolamine and methysergide but is unaffected by the intrathecal administration of either phentolamine or methysergide alone (Randich and Aicher, 1988; Ren et al., 1988).

These data are consistent with the view that the NTS may play an important role in the modulation of pain and operate by integrating visceral afferent input from the cardiopulmonary region with spinopetal systems of descending inhibition (see Randich and Maixner, 1984a; Menetrey and Basbaum, 1987). The purpose of the present studies was to characterize inhibition of the tail-flick reflex produced by microinjections of glutamate into the NTS and the regions ventral to the NTS. The primary ventral region of investigation was the nucleus reticularis ventralis (NRV) according to the nomenclature of Newman (1985), but sites of microinjection also included the nucleus reticularis parvocellularis (NRPC) in rostral regions, the dorsal motor nucleus of the vagus (DMV), and hypoglossal nucleus. Glutamate was chosen as a stimulus since the secondary projection cells of vagal afferents arise from this region and glutamate is believed to activate only cell bodies (Johnson, 1972; Goodchild et al., 1982). The use of glutamate thereby alleviates problems of interpretation associated with previous studies using electrical stimulation, in which both cells and fibers of passage are activated. In Experiment 1, various sites of microinjection of glutamate in the medulla were assessed for the capacity to produce antinociception; Experiment 2 established dose/volume-effect functions for glutamate; Experiment 3 characterized the nature of spinopetal systems mediating antinociception produccd by glutamate microinjections in these regions through the use of 
intrathecal administration of receptor antagonists; and Experiment 4 assessed whether feedback from cardiovascular pressor responses evoked by the microinjection of glutamate contributed to the antinociception.

\section{Materials and Methods}

Subjects. The subjects were 139 experimentally naive, male SpragueDawley rats obtained from Hormone Assay Laboratories in Chicago. The rats were individually housed in wire-mesh cages under a $12: 12 \mathrm{hr}$ light-dark cycle. Food and water were available on an ad libitum basis.

Apparatus. A radiant heat stimulus provided by a projector lamp housed in a metal casing was used to evoke the nociceptive tail-flick reflex and has been described elsewhere (Randich and Aicher, 1988). Surface temperature of the tail increased from an ambient level of $38^{\circ} \mathrm{C}$. to a terminal level of $52^{\circ} \mathrm{C}$ over a $10 \mathrm{sec}$ period with approximately a $1.5^{\circ} \mathrm{C} / \mathrm{sec}$ increase in temperature.

Arterial blood pressure and heart rate were recorded via the pulse pressure signal provided by a Century pressure transducer and an APPLE II + computer.

General surgical techniques and testing. Each rat was anesthetized with pentobarbital sodium $(50 \mathrm{mg} / \mathrm{kg}$, i.p.). A catheter was implanted in the left common carotid artery for recording of arterial blood pressure, and a tracheostomy was performed. The rat was then placed in a stereotaxic device and the medulla was exposed. The microinjector was placed in position in the medulla. Each rat received baseline tail-flick test trials in the lightly anesthetized state. The lightly anesthetized state was maintained by i.a. pentobarbital supplements of $2.50 \mathrm{mg}$ approximately every $45 \mathrm{~min}$ after the initial injection. Tail position was varied randomly, and the same position was not heated twice in succession so as to prevent damage to the tail. The tail-flick latency was defined as the interval between the onset of heating and the withdrawal of the tail from the heat source. If a tail-flick reflex did not occur within $10 \mathrm{sec}$ of heat onset, then heat stimulation automatically terminated. A $10 \mathrm{sec}$ cut-off time is 2.5-3 times the normal response latency and does not result in tissue damage to the tail. A baseline latency was obtained when responses were stable and between 3-4 sec.

Experiment 1: tracking. In Experiment 1, 44 rats received microinjections of glutamate into the NTS from -13.24 to $-14.30 \mathrm{~mm}$ from bregma, and into sites ventral to the NTS. Glutamate $(100 \mathrm{~mm}$ in sterile saline) was microinjected in a volume of of $0.5 \mu \mathrm{l}(50 \mathrm{nmol})$ through a 33 gauge $(0.20$ O.D.) injection cannula inserted into and extending 1$3 \mathrm{~mm}$ beyond the end of a guide cannula. The guide cannula was never inserted into medullary tissue. The injection was delivered over a 30 sec period, and the injection cannula was left in place. The progress of the microinjection was monitored by observing travel of an air bubble in a length of calibrated PE 10 tubing connecting the injection cannula with a $5 \mu$ l syringe. Tail-flick test trials were administered 1, 2, 3, 4, and $5 \mathrm{~min}$ after the start of the injection, but only data for the $1 \mathrm{~min}$ test trial are reported since most effects dissipated by the 2 min test trial. Similar, short-lasting antinociceptive effects of glutamate microinjected into the brain stem have been reported with these volumes and concentrations in other studies (Aimone and Gebhart, 1986; Janss and Gebhart, 1987). In each of 25 rats, 4 microinjections of glutamate were made in the dorsal-ventral plane with the initial microinjection starting $0.3-0.8 \mathrm{~mm}$ ventral to the surface of the medulla; each succeeding microinjection was made $0.5 \mathrm{~mm}$ more ventral to the preceding microinjection. Each microinjection was separated by a 20 min recovery period, and new baseline tail-flick latencies were established prior to each microinjection. In an additional 19 rats, only a single microinjection was delivered. In almost all cases, rats required artificial ventilation following the microinjection of glutamate. Artificial ventilation produced no apparent changes in the tail-flick lantency, but the decreases in arterial blood pressure at the time of change from self-generated to artificial respiration would be less had it been possible to use artificial respiration throughout. However, these arterial blood pressure values were not used in any of the data analyses, and the values reported 1 min after microinjection of glutamate represent the animal being sustained on artificial respiration for approximately $45 \mathrm{sec}$.

Experiment 2: dose/volume. In Experiment 2, 12 rats received microinjections of glutamate into either the depressor area of the NTSDMV and the immediate ventral border of this region $(n=6)$ located -13.30 to $-13.80 \mathrm{~mm}$ from bregma or the pressor area in the NRV $(n=6)$ in the same rostral-caudal plane (1.5 mm ventral to the dura). Each rat received 500,300 , and $100 \mathrm{nl}$ of glutamate $(50,30$, and 10 nmol, respectively), and a control injection of $500 \mathrm{nl}$ of isotonic saline vehicle. Tail-flick test trials were presented as previously described. Baseline tail-flick latencies were obtained prior to each microinjection of glutamate, with a 20 min interval between each microinjection.

Experiment 3: intrathecal receptor antagonists. Seventy-seven anesthetized rats had PE10 catheters inserted in the subarachnoid space to the region of the lumbar enlargement using the method described by Yaksh and Rudy (1976). The microinjector was then placed in either a depressor $(n=38)$ or pressor $(n=39)$ region $(-13.68$ to $-13.80 \mathrm{~mm}$ from bregma), and $500 \mathrm{nl}$ of saline was microinjected to determine if the vehicle exerted an effect. Only 2 rats showed any substantial influence of saline on the tail-flick reflex and were replaced. Each rat then received microinjection of $50 \mathrm{nmol}$ of glutamate $(500 \mathrm{nl})$ into the same region to establish that it increased the tail-flick reflex latency at least $50 \%$ above baseline values; $70 / 77$ rats manifested a $10 \mathrm{sec}$ cut-off on this test. Three groups of rats $(n=12 /$ group with half the rats showing depressor and half the rats showing pressor responses assigned to each group) then received intrathecal administration of a receptor antagonist. The intrathecal injections were $30 \mu \mathrm{g}$ of either (1) naloxone $\mathrm{HCl}$ (Sigma), (2) methysergide maleate (Sandoz), or (3) phentolamine mesylate (CibaGeigy). Intrathecal injections were $7.5 \mu \mathrm{l}$ followed by an $8 \mu \mathrm{l}$ saline flush. At $10 \mathrm{~min}$ after administration of the receptor antagonist, each rat received a second, identical microinjection of glutamate. A group of control rats $(n=12$ with half pressor and half depressor responses to glutamate) received no intrathecal antagonist prior to the second test of glutamate.

Rats that received either methysergide or phentolamine alone in the aforementioned tests then received the other receptor antagonist, resulting in a total of 12 rats with the combination of phentolamine (30 $\mu \mathrm{g})$ and methysergide $(30 \mu \mathrm{g})$ in the lumbar region. A third microinjection of glutamate was then administered $5 \mathrm{~min}$ after the second receptor antagonist was given. The control rats received a third microinjection of glutamate in the absence of any receptor antagonist and temporally matched to the microinjections of glutamate in the experimental rats.

Finally, since the combined administration of $30 \mu \mathrm{g}$ each of phentolamine and methysergide resulted in antagonism of the antinociception, 29 rats (15 pressor and 14 depressor) were tested with combinations of these drugs in $7.5 \mu \mathrm{g}(n=14)$ and $15 \mu \mathrm{g}$ doses $(n=15)$ to assess dose dependency.

Experiment 4: hexamethonium. Experiment 4 assessed whether the peripheral pressor response evoked by microinjection of glutamate into the NRV contributed to the antinociception by feedback through the baroreceptor reflex arc. Rats $(n=6)$ received microinjections of glutamate $(50 \mathrm{nmol}$ in $500 \mathrm{nl})$ into the NRV to establish that the treatment resulted in both inhibition of the tail-flick reflex and a pressor response. Each rat then received $20 \mathrm{mg} / \mathrm{kg}$, i.a., of hexamethonium bromide following which the capacity of glutamate to inhibit the tail-flick reflex in the absence of an increase in arterial blood pressure was retested.

Data analysis. In Experiment 1, each tail-flick latency was converted to a tail-flick index (TFI) by the equation [(trial latency - baseline latency $) /(10 \mathrm{sec}-$ baseline latency $)] \times 100$ for ease of data presentation. Cardiovascular effects were expressed as the percentage change from baseline in both Experiments 1 and 2 for ease of data presentation. Actual cardiovascular values are reported in Experiments 3 and 4, but statistical analyses of the cardiovascular data in Experiment 3 were performed on percentage change measures since baselines differed between groups. One-way and randomized blocks ANOVAs were used for all data analyses. Alpha was 0.05 .

Histology. All injection sites were histologically verified either by lesions made with a stimulating electrode $(500 \mu \mathrm{A}$ DC for $3 \mathrm{sec})$ or visualizing the microinjector tract, and staining coronal brain sections $(40 \mu \mathrm{m})$ with cresyl violet. Intrathecal placements were verified by injecting Fast green dye through the catheter and inspecting both the location of the dye and the integrity of the spinal cord.

\section{Results}

\section{Tracking: tail-flick}

Figure 1 presents 113 sites of microinjection of glutamate in which the medulla is illustrated in 6 sections extending 1.06 $\mathrm{mm}$ in the rostral-caudal plane. The symbols designate sites of microinjection that resulted in no effect on the tail-flick reflex (open triangles, $<20 \%$ elevation in TFI), partial inhibition of the reflex (open circles, $21-99 \%$ elevation in the TFI), or com- 
TAIL-FLICK

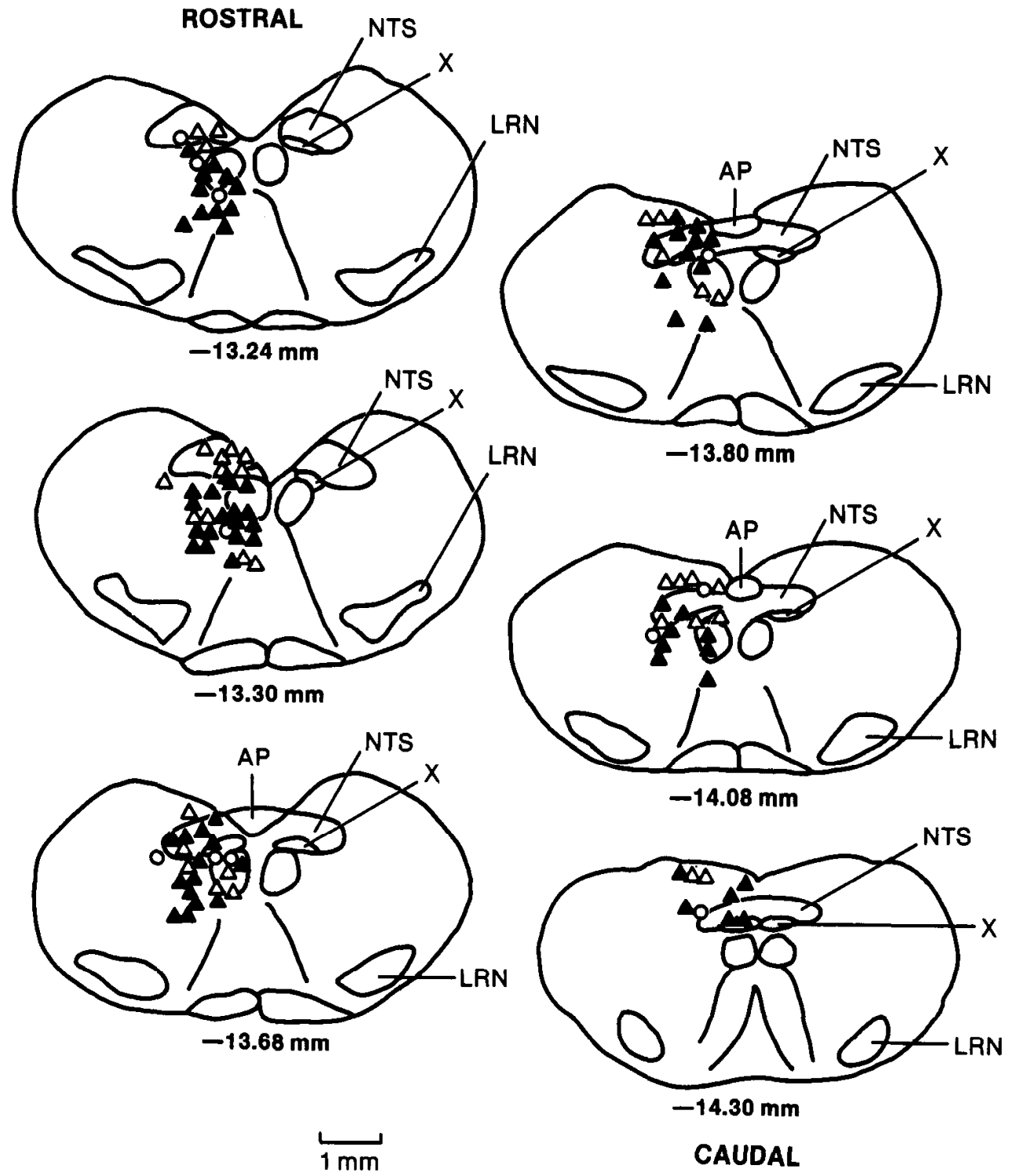

Figure 1. Sites in which microinjection of glutamate exerted either no effect (TFI $<20, \Delta$ ), a partial effect (TFI of 21-99, O), or complete inhibition of the tail-flick reflex (TFI of 100, $\Delta$ ). plete inhibition of the reflex (solid triangles, $100 \%$ TFI value; the cut-off latency of $10 \mathrm{sec}$ ). In general, microinjections of glutamate into rostral NTS were without effect, whereas more caudal and medial injections into the NTS resulted in complete inhibition of the tail-flick reflex. Microinjections of glutamate into the NRV also resulted in the production of antinociception. Antinociception from the NRV sites could be obtained throughout the rostral-caudal axis of the NRV sampled in this study, but the most consistent effects appeared to be with rostral microinjections where the NRV merges into the NRPC. The group mean TFI changes at the $1 \mathrm{~min}$ test trial were $6.36 \pm 1.48 \%$ (no effect sites) and $36.25 \pm 4.99 \%$ (partial inhibition sites).

\section{Tracking: arterial blood pressure}

Figure 2 presents the sites of microinjection and changes in arterial blood pressure resulting from microinjection of glutamate: no effect (open circles, $<10 \%$ increase or decrease in arterial blood pressure), depressor response (open triangles, $>10 \%$ decrease in arterial blood pressure), or pressor response (solid triangles, $>10 \%$ increase in arterial blood pressure). In all the cases in which glutamate was microinjected into the NTS, arterial blood pressure either decreased or remained at baseline levels (see below). Depressor responses were also evoked on the ventral border of the NTS and in the NRPC, but acute pressor responses were also observed, particularly with more ventral microinjections in the NRV. It should also be noted that pressor responses were obtained from microinjections that impinged on the hypoglossal nucleus. The mean change in arterial blood pressure at the $1 \mathrm{~min}$ test trial was $1.43 \pm 0.88 \%$ (no effect sites); $-34.67 \pm 2.00 \%$ (depressor sites); and $39.00 \pm 2.51 \%$ (pressor sites). Data were collected for $5 \mathrm{~min}$ postinjection, but in almost all cases arterial blood pressure returned to baseline levels within 2-3 min. It should be emphasized that depressor responses were obtained throughout the rostral-caudal extent of the NTS, despite the previous observation that antinociception could only be obtained in the caudal regions of NTS. Pressor responses 


\section{ARTERIAL BLOOD PRESSURE}

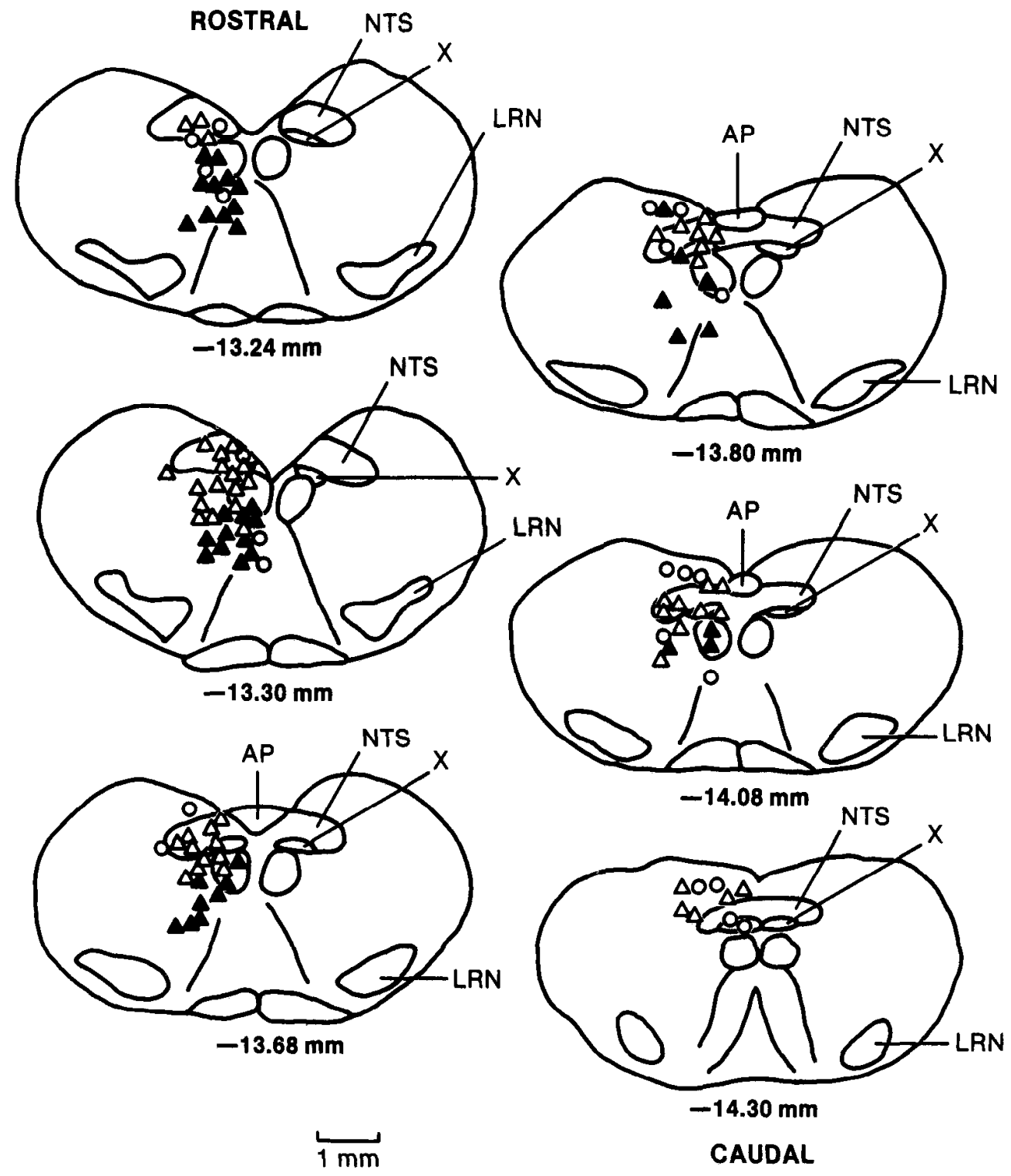

Figure 2. Sites in which microinjections of glutamate exerted either no effect $(<10 \%$ change from baseline, $O)$, a depressor response $(>10 \%$ decrease from baseline, $\Delta$ ), or a pressor response $(>10 \%$ increase from baseline, $\Delta)$.

were obtained throughout the rostral-caudal extent of NRV. Finally, in virtually all rats, heart rate decreased slightly in response to microinjections of glutamate regardless of whether depressor or pressor responses were obtained. The mean changes in heart rate were $-5.64 \pm 1.90 \%,-15.11 \pm 1.50 \%$, and -17.00 $\pm 2.89 \%$ for the no effect, depressor, and pressor sites, respectively. Figures 3 and 4 present examples of sites of microinjection in the NTS and the NRV, respectively.

\section{Dose/volume functions}

Experiment 2 established dose/volume-effect functions for glutamate when microinjected into either sites in the NTS that resulted in a depressor response or sites in the NRV that resulted in a pressor response. Figure 5 presents the outcomes of these manipulations. The upper left panel of Figure 5 shows significant increases in the tail-flick latency as a function of the dose/volume of glutamate microinjected $(F 3,15=13.95)$; the $500 \mathrm{nl}$
(50 nmol) microinjection produced inhibition of the tail-flick reflex to the $10 \mathrm{sec}$ cut-off in all rats. Saline administration in a volume equal to the largest volume of glutamate $(500 \mathrm{nl})$ resulted in no inhibition of the tail-flick reflex or apnea. The bottom left panel of Figure 5 indicates that microinjection of glutamate into the NTS resulted in depressor responses that monotonically increased with dose, but an ANOVA indicated that the magnitude of the depressor response did not significantly increase with dose/volume $(F 3,15=1.64)$. This volume of saline vehicle $(500 \mathrm{nl})$ consistently decreased arterial blood pressure during the injection procedure to levels comparable to those produced by $50 \mathrm{nmol}$ of glutamate. However, arterial blood pressure returned to near-normal levels by the $1 \mathrm{~min}$ assessment trial. All sites of microinjection of glutamate producing a decrease in arterial blood pressure were between -13.68 to $-13.80 \mathrm{~mm}$ from bregma, -0.8 to $1.2 \mathrm{~mm}$ ventral to the dura (some depressor sites were just ventral to the NTS-DMV 


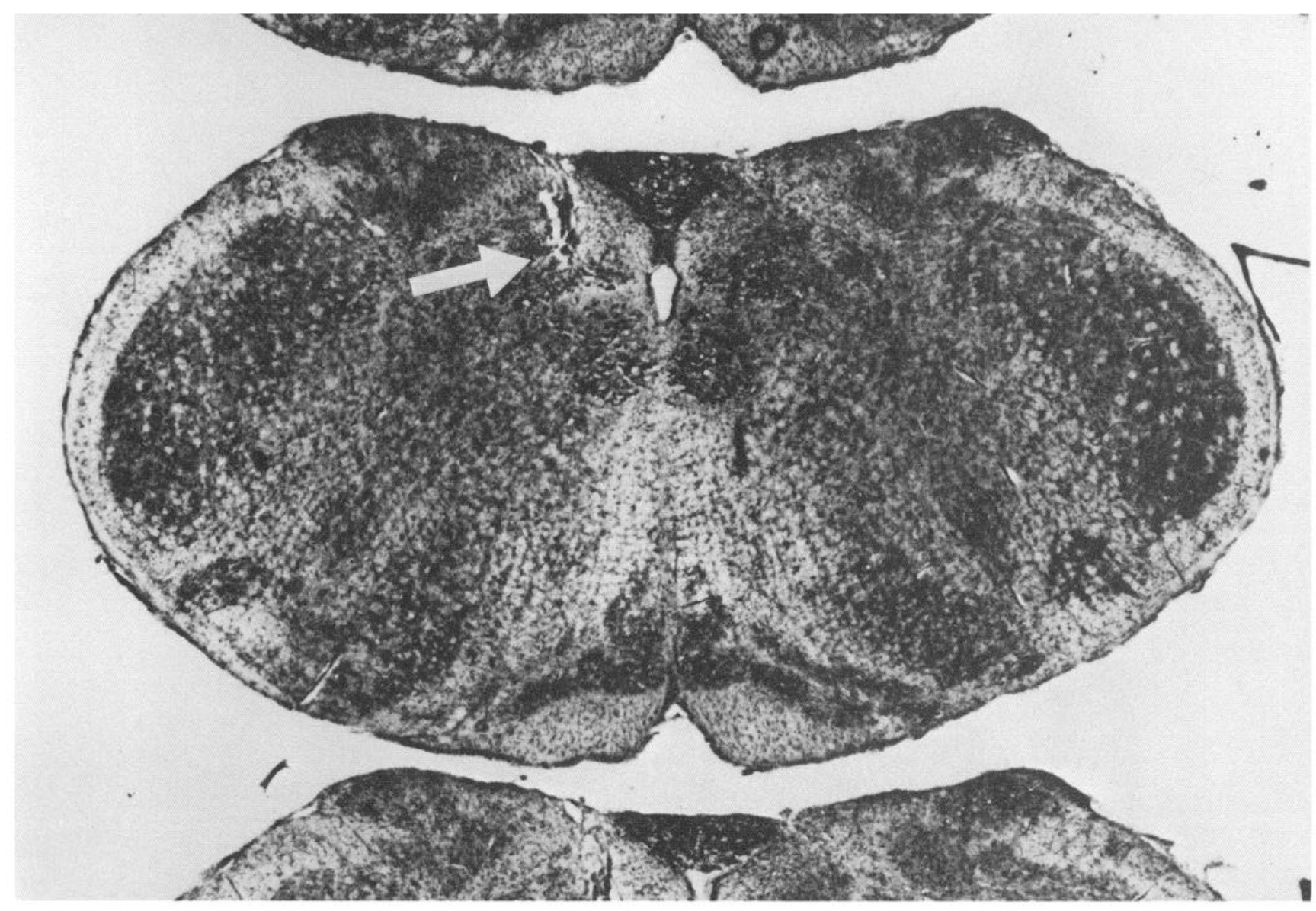

Figure 3. Example of a site of microinjection of glutamate in the NTS at the level of the area postrema.

border as shown in Fig. 1), and $0.8-1.0 \mathrm{~mm}$ lateral to the midline.

The right panels of Figure 5 indicate significant dose/volumedependent increases in tail-flick latencies $(F 5,15=24.87)$ and significant dose-dependent pressor responses $(F 5,15=5.40)$ following microinjections of glutamate into the NRV. In terms of the tail-flick reflex, both 30 and $50 \mathrm{nmol}$ glutamate increased tail-flick latencies to the 10 -sec cut-off in all rats at the $1 \mathrm{~min}$ test trial, although the $50 \mathrm{nmol}$ dose resulted in a marginally longer-lasting inhibition over the total $5 \mathrm{~min}$ assessment period (not shown). As was observed in Experiment 1, bradycardia occurred in both the depressor and pressor groups, and heart rate systematically decreased as a function of the dose/volume of glutamate. However, changes in heart rate were not statistically significant, and the maximal mean percentage decrease observed was $16 \%$ below baseline levels. All sites of microinjection of glutamate producing an increase in arterial blood pressure were between -13.30 to $-13.80 \mathrm{~mm}$ from bregma, -1.5 to $-2.0 \mathrm{~mm}$ from the dura, and $0.8-1.0 \mathrm{~mm}$ lateral to the midline.

\section{Intrathecal receptor antagonists}

Tables 1 and 2 summarize the effects of intrathecal administration of receptor antagonists on inhibition of the tail-flick reflex produced by glutamate. The data are grouped by sites that produced either depressor (NTS) or pressor responses (NRV $-1.5 \mathrm{~mm}$ ventral to the dura) during the pretest with microin- jection of glutamate. Saline control data are also presented on the basis of depressor (Table 1) and pressor sites (Table 2). It should also be noted that the groups receiving combined administration of $30 \mu \mathrm{g}$ each of phentolamine and methysergide have a separate control group that equates the number of microinjections of glutamate (i.e., 3).

In general, microinjection of saline vehicle $(500 \mathrm{nl})$ resulted in either no or small increases in the latency of the tail-flick reflex. During the pre-intrathecal test with glutamate (not presented), tail-flick latencies were significantly increased above saline control values (70/77 rats showed a 10 sec cut-off), and none of the groups identified in Tables 1 and 2 differed significantly in the magnitude of the response to glutamate. During the tests of the single receptor antagonists ( $30 \mu \mathrm{g}$ of either naloxone, methysergide, or phentolamine), tail-flick latencies were significantly increased by microinjection of glutamate relative to baselines, and a 1-way ANOVA revealed no significant attenuation of the response relative to either the glutamate control group receiving its second glutamate microinjection or pre-intrathecal glutamate control values (not shown). Thus, administration of naloxone, phentolamine, or methysergide alone did not attenuate glutamate-induced inhibition of the tail-flick reflex compared with control values in any of the groups in either the depressor or pressor conditions.

In contrast, the combined administration of phentolamine and methysergide resulted in a dose-dependent attenuation of the antinociceptive effect of microinjection of glutamate in rats 


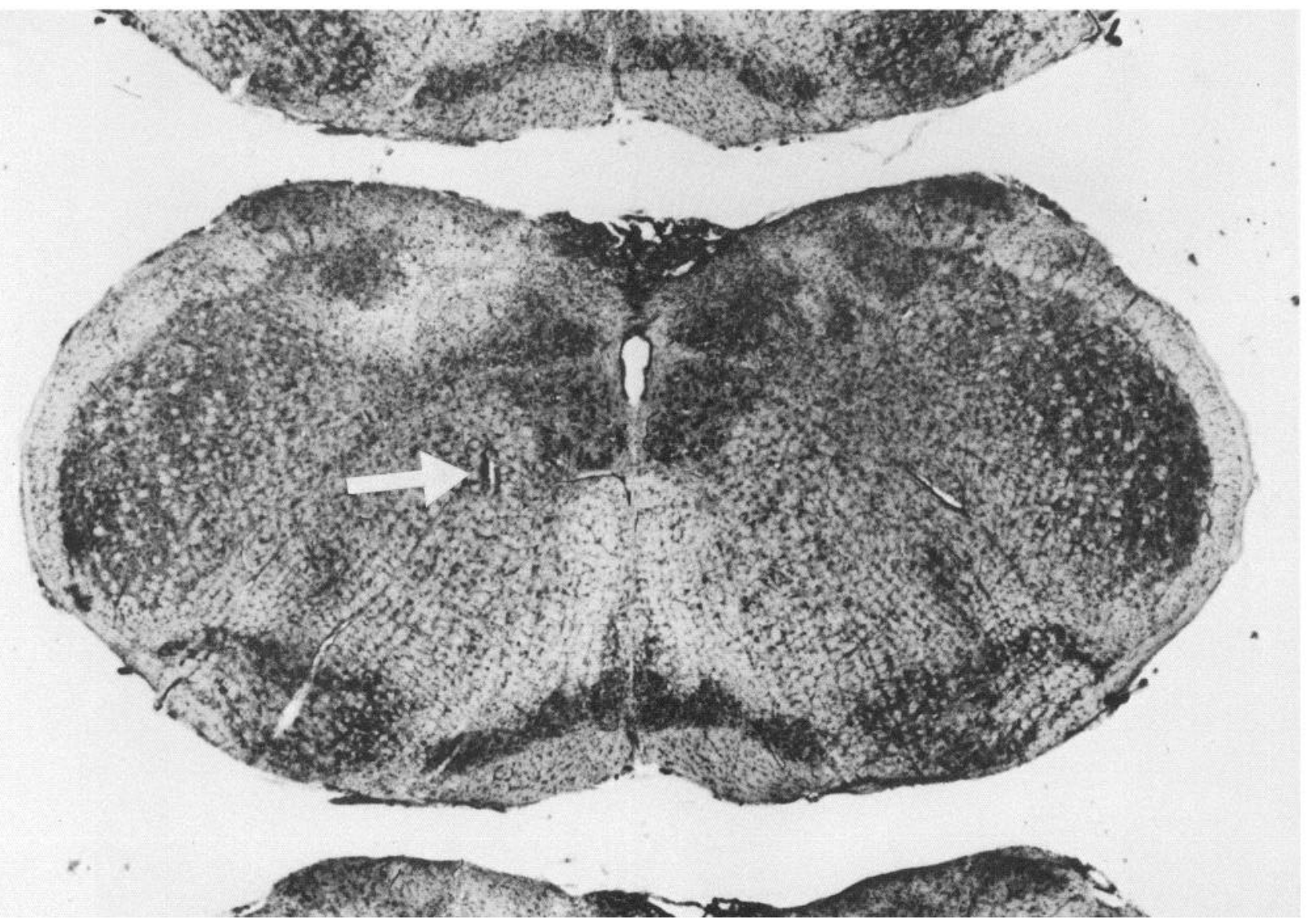

Figure 4. Example of a site of microinjection of glutamate in the NRV at the level of the area postrema near the border of the hypoglossal nucleus.

showing either a depressor or a pressor response. In the combined $30 \mu \mathrm{g}$ conditions, tail-flick latencies obtained following microinjection of glutamate did not significantly differ from their corresponding tail-flick latencies obtained following microinjection of saline, indicating complete antagonism of the antinociception. In the combined $7.5 \mu \mathrm{g}$ conditions, tail-flick latencies did not significantly differ from their glutamate control values, indicating no antagonism of the antinociception. In the combined $15 \mu \mathrm{g}$ conditions, tail-flick latencies in the depressor condition were significantly decreased relative to their glutamate control values, whereas tail-flick latencies in the pressor condition were nonsignificantly decreased relative to their glutamate control values.

The remainder of these tables present cardiovascular responses observed at the $1 \mathrm{~min}$ test trial following microinjection of glutamate in the presence of the intrathecal antagonists. ANOVAs indicated no significant between-groups differences in the magnitude of the depressor response evoked by microinjection of glutamate during either the second or third microinjection. ANOVAs of the pressor data also failed to reveal significant between-groups differences with the exception that the group receiving combined administration of $30 \mu \mathrm{g}$ phentolamine and $30 \mu \mathrm{g}$ methysergide showed a significantly smaller pressor response than the pressor control condition. ANOVAs failed to reveal any significant between-groups differences in the percentage change in heart rates for either depressor or pressor conditions. All sites of microinjection producing depressor re- sponses were between -13.30 to $-14.08 \mathrm{~mm}$ from bregma either in the NTS-DMV region or on the ventral border of this region. All sites of microinjection producing pressor responses were located between -13.24 to $-14.30 \mathrm{~mm}$ from bregma.

\section{Hexamethonium blockade of the pressor response}

Experiment 4 assessed whether blocking the peripheral pressor response evoked by microinjection of glutamate into the NRV would attenuate the antinociceptive effect, since the magnitudes of both the pressor response and antinociception were significantly reduced by the intrathecal administration of $30 \mu \mathrm{g}$ each of phentolamine and methysergide in Experiment 3, and since it has been demonstrated that elevations in arterial blood pressure produced by administration of phenylephrine can produce modest increases in tail-flick latencies (Randich and Maixner, 1984a). In the glutamate pretest, microinjection of glutamate (1) increased the latency of the tail-flick reflex from a group mean baseline of $3.82 \pm 0.20 \mathrm{sec}$ to the $10 \mathrm{sec}$ cut-off in all 6 rats; (2) increased arterial blood pressure from a group mean baseline of $100 \pm 7$ to $162 \pm 7 \mathrm{~mm} \mathrm{Hg}$; and (3) decreased heart rate from a group mean baseline of $339 \pm 13$ to $305 \pm 16$ beats/ min. After intra-arterial administration of $20 \mathrm{mg} / \mathrm{kg}$ of hexamethonium, microinjection of glutamate (1) increased the latency of the tail-flick reflex from a group mean baseline of 4.08 \pm 0.15 to the $10 \mathrm{sec}$ cut-off in all rats; (2) decreased arterial blood pressure from a group mean baseline of $53 \pm 5$ to $50 \pm$ $9 \mathrm{~mm} \mathrm{Hg}$; and (3) increased heart rate from a group mean 

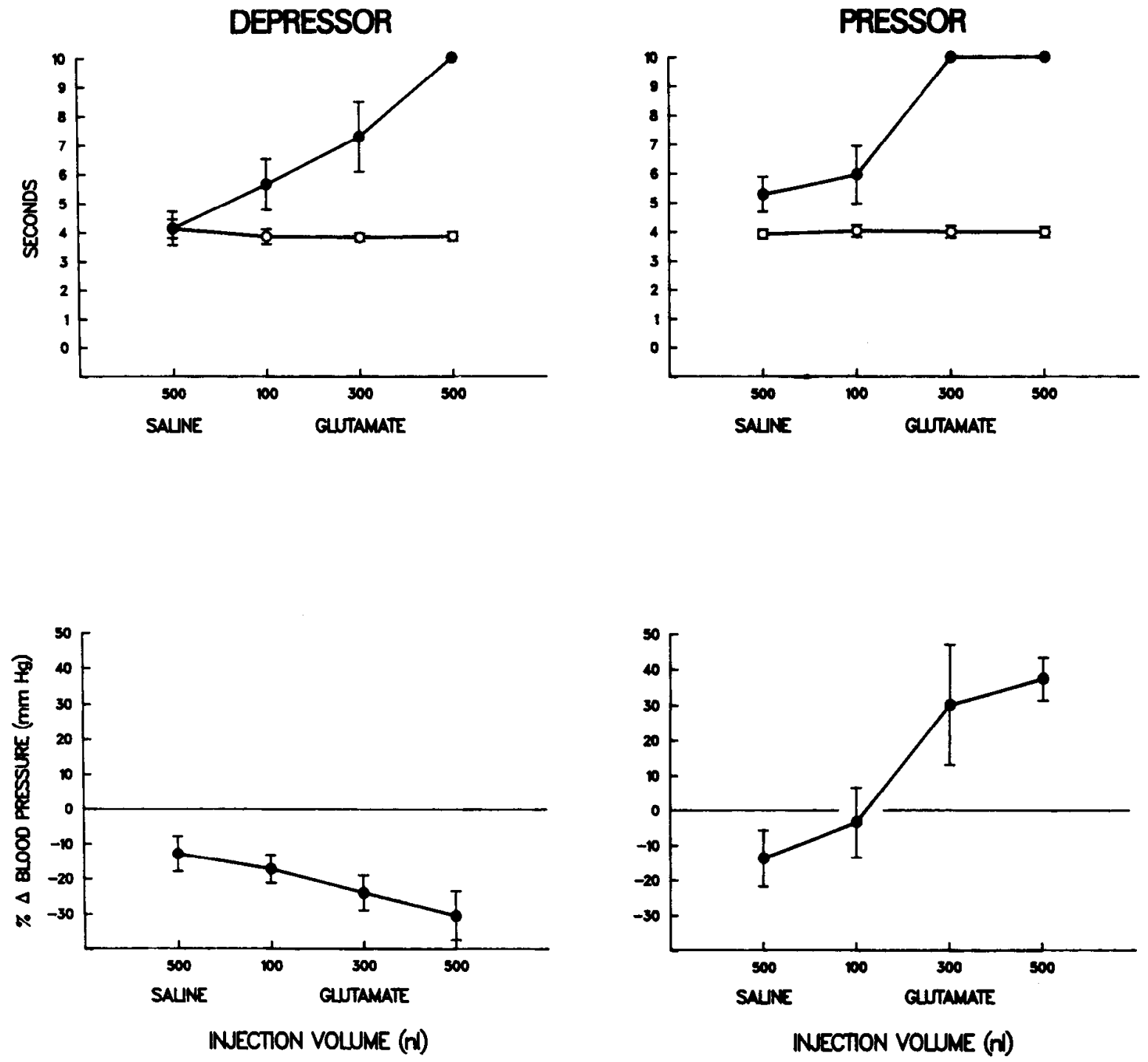

Figure 5. Dose/volume-effect functions for glutamate and saline vehicle using inhibition of the tail-flick reflex and arterial blood pressure as response measures. Volumes of saline vehicle and glutamate are given along the abscissae. Injection sites were in the depressor region of the NTS (left panels) or the pressor regions of the NRV (right panels). Open circles indicate mean baseline tail-flick latencies; closed symbols, values obtained $1 \mathrm{~min}$ after microinjection of glutamate or saline.

baseline of $288 \pm 9$ to $335 \pm 27$ beats $/ \mathrm{min}$. All sites of microinjection of glutamate were between -13.30 to $-13.68 \mathrm{~mm}$ from bregma, $1.5 \mathrm{~mm}$ ventral to the dura, and 0.8 to $1.0 \mathrm{~mm}$ lateral to the midline. Thus, hexamethonium resulted in complete blockade of the pressor response evoked by glutamate microinjectcd in the NRV, but inhibition of the tail-flick reflex resulting from the glutamate microinjection was not attenuated.

\section{Discussion}

Microinjections of glutamate into the caudal NTS, but not rostral NTS resulted in inhibition of the tail-flick reflex. Hypotension and mild bradycardia were also evoked by microinjections throughout the entire rostral-caudal extent of the NTS examined in the present study. The antinociceptive responses were significantly increased as a function of the dose/volume of glutamate injected into the caudal NTS, and the magnitude of the depressor response increased monotonically with drug dose/ volume. Microinjections of glutamate into the region ventral to the NTS and traversing a large rostral-caudal extent also re- sulted in inhibition of the tail-flick reflex, but they were consistently accompanied by pressor responses, particularly with sites of microinjection in the NRV. These responses were dose/ volume dependent, and the antinociception obtained with microinjections of glutamate resulting in a pressor response could be achieved with a lower dose of glutamate than when they produced depressor responses.

The ability to obtain antinociception, hypotension, and bradycardia from the caudal NTS suggests that this region may serve an important role in integrating information from visceral structures in the modulation of somatosensory input. Cardiopulmonary afferents terminate in this region of the NTS (Kalia and Sullivan, 1982), and numcrous studies have demonstrated that peripheral activation of cardiopulmonary afferents also results in antinociception, hypotension, and bradycardia of comparable magnitude to those obtained with glutamate microinjected into the caudal NTS (see, for example, Randich and Aicher, 1988; Ren et al., 1988). Of equal importance is the demonstration of antinociception, hypertension, and bradycardia evoked 


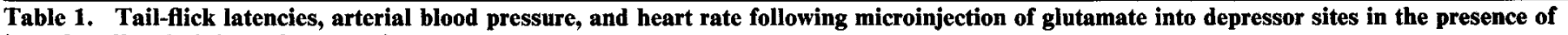
intrathecally administered antagonists

\begin{tabular}{|c|c|c|c|c|c|c|c|c|c|c|}
\hline \multirow[b]{3}{*}{ Group } & \multirow[b]{3}{*}{$N$} & \multirow{3}{*}{$\begin{array}{l}\text { Dose } \\
(\mu \mathrm{g})\end{array}$} & \multirow{2}{*}{\multicolumn{2}{|c|}{$\begin{array}{l}\text { Saline vehicle } \\
\text { tail-flick (sec) }\end{array}$}} & \multicolumn{6}{|c|}{ Glutamate and IT antagonist } \\
\hline & & & & & \multicolumn{2}{|c|}{$\overline{\text { Tail-flick (sec) }}$} & \multicolumn{2}{|c|}{ Blood pressure $(\mathrm{mm} \mathrm{Hg})$} & \multicolumn{2}{|c|}{ Heart rate (beats/min) } \\
\hline & & & Baseline & Test & Baseline & Test & Baseline & Test & Baseline & Test \\
\hline Control & 6 & - & $3.43 \pm 0.31$ & $3.85 \pm 0.49$ & $3.10 \pm 0.38$ & $9.29 \pm 0.70$ & $123 \pm 6$ & $95 \pm 11$ & $317 \pm 14$ & $296 \pm 9$ \\
\hline Naloxone & 6 & 30 & $3.68 \pm 0.22$ & $3.69 \pm 0.44$ & $3.54 \pm 0.30$ & $8.98 \pm 1.01$ & $128 \pm 5$ & $102 \pm 10$ & $330 \pm 48$ & $284 \pm 44$ \\
\hline Methysergide & 6 & 30 & $3.06 \pm 0.42$ & $3.42 \pm 0.56$ & $2.85 \pm 0.54$ & $7.98 \pm 1.37$ & $127 \pm 8$ & $104 \pm 11$ & $351 \pm 17$ & $313 \pm 9$ \\
\hline Phentolamine & 6 & 30 & $3.33 \pm 0.49$ & $4.02 \pm 0.76$ & $3.24 \pm 0.32$ & $7.72 \pm 1.09$ & $100 \pm 6$ & $94 \pm 9$ & $335 \pm 14$ & $321 \pm 7$ \\
\hline $\begin{array}{l}\text { Phentolamine/ } \\
\text { methysergide }\end{array}$ & 7 & 7.5 & $3.74 \pm 0.16$ & $3.95 \pm 0.29$ & $3.51 \pm 0.15$ & $8.63 \pm 0.89$ & $131 \pm 8$ & $116 \pm 13$ & $365 \pm 17$ & $318 \pm 13$ \\
\hline $\begin{array}{l}\text { Phentolamine/ } \\
\text { methysergide }\end{array}$ & 7 & 15 & $3.54 \pm 0.18$ & $3.25 \pm 0.21$ & $2.92 \pm 0.19$ & $5.36 \pm 1.22^{a}$ & $119 \pm 8$ & $104 \pm 19$ & $352 \pm 20$ & $298 \pm 23$ \\
\hline $\begin{array}{l}\text { Phentolamine/ } \\
\text { methysergide } \\
\text { Control for } \\
\text { phentolamine/ } \\
\text { methysergide, }\end{array}$ & 12 & 30 & $3.20 \pm 0.31$ & $3.72 \pm 0.46$ & $2.72 \pm 0.30$ & $3.69 \pm 0.64^{a}$ & $104 \pm 5$ & $98 \pm 5$ & $337 \pm 14$ & $328 \pm 10$ \\
\hline
\end{tabular}

Tail-flick latencies for the saline vehicle test are also presented. "Test" signifies the value 1 min after microinjection. SEM are denoted for each mean.

a Significantly different from control $(p<0.05)$.

by glutamate microinjected in regions ventral to the NTS. A variety of evidence would indicate that these effects are due to stimulation of cell bodies in this region and not a consequence of some other factor (for example, spread of the injectate to the $\mathrm{C} 1$ region, where similar responses can be evoked). First, Minson et al. (1987) obtained pressor responses following microinjections of glutamate $(10 \mathrm{nmol}$ in $100 \mathrm{nl})$ into either regions comparable to those of the present study or more ventral sites in the medial medullary B3 cell group. They showed that the pressor responses obtained from the $\mathrm{B} 3$ region were significantly attenuated by prior intracerebroventricular administration of 5,7-dihydroxytryptamine $(5,7-\mathrm{DHT})$, but that the pressor re- sponses obtained from sites of microinjection corresponding to sites of the present study in the NRV were only nonsignificantly attenuated by this treatment. In addition, they showed that either unilateral electrolytic lesions of the $\mathrm{C} 1$ region or pretreatment with the phenylethanolamine- $N$-methyltransferase (PNMT) inhibitor LY134046 did not affect the pressor responses from either of these 2 pressor regions. Second, Leibstein et al. (1985) showed a band of vasoactive intestinal peptide (VIP)-like reactive neurons by immunohistochemistry that correspond extremely well with sites of microinjection in the present study that resulted in pressor responses. Thus, it may be the case that the glutamate microinjections of the present study

Table 2. Tail-flick latencies, arterial blood pressure, and heart rate following microinjection of glutamate into pressor sites in the presence of intrathecally administered antagonists

\begin{tabular}{|c|c|c|c|c|c|c|c|c|c|c|}
\hline \multirow[b]{3}{*}{ Group } & \multirow[b]{3}{*}{$N$} & \multirow{3}{*}{$\begin{array}{l}\text { Dose } \\
(\mu \mathrm{g})\end{array}$} & \multirow{2}{*}{\multicolumn{2}{|c|}{$\begin{array}{l}\text { Saline vehicle } \\
\text { tail-flick (sec) }\end{array}$}} & \multicolumn{6}{|c|}{ Glutamate and IT antagonist } \\
\hline & & & & & \multicolumn{2}{|c|}{ Tail-flick (sec) } & \multicolumn{2}{|c|}{ Blood pressure (mm Hg) } & \multicolumn{2}{|c|}{ Heart rate (beats/min) } \\
\hline & & & Baseline & Test & Baseline & Test & Baseline & Test & Baseline & Test \\
\hline Control & 6 & - & $3.99 \pm 0.09$ & $4.41 \pm 0.32$ & $3.77 \pm 0.13$ & $8.97 \pm 1.03$ & $111 \pm 12$ & $154 \pm 13$ & $340 \pm 13$ & $300 \pm 33$ \\
\hline Naloxone & 6 & 30 & $3.79 \pm 0.14$ & $3.86 \pm 0.18$ & $3.71 \pm 0.11$ & $10.00 \pm 0.00$ & $112 \pm 3$ & $157 \pm 12$ & $346 \pm 9$ & $314 \pm 21$ \\
\hline Methysergide & 6 & 30 & $3.27 \pm 0.35$ & $3.17 \pm 0.40$ & $3.48 \pm 0.24$ & $8.07 \pm 1.24$ & $129 \pm 8$ & $152 \pm 6$ & $379 \pm 16$ & $331 \pm 19$ \\
\hline Phentolamine & 6 & 30 & $3.47 \pm 0.20$ & $3.88 \pm 0.16$ & $3.21 \pm 0.16$ & $8.86 \pm 1.13$ & $94 \pm 4$ & $152 \pm 15$ & $313 \pm 17$ & $342 \pm 14$ \\
\hline $\begin{array}{l}\text { Phentolamine/ } \\
\text { methysergide }\end{array}$ & 7 & 7.5 & $3.67 \pm 0.10$ & $4.84 \pm 0.43$ & $3.50 \pm 0.28$ & $9.06 \pm 0.93$ & $121 \pm 6$ & $184 \pm 4$ & $322 \pm 14$ & $298 \pm 23$ \\
\hline $\begin{array}{l}\text { Phentolamine/ } \\
\text { methysergide }\end{array}$ & 8 & 15 & $3.60 \pm 0.27$ & $3.96 \pm 0.38$ & $3.15 \pm 0.21$ & $6.69 \pm 1.33$ & $101 \pm 9$ & $162 \pm 8$ & $334 \pm 18$ & $351 \pm 21$ \\
\hline $\begin{array}{l}\text { Phentolamine/ } \\
\text { methysergide }\end{array}$ & 12 & 30 & $3.37 \pm 0.19$ & $3.53 \pm 0.23$ & $3.26 \pm 0.17$ & $4.82 \pm 0.55^{a}$ & $103 \pm 4$ & $115 \pm 11^{a}$ & $342 \pm 14$ & $351 \pm 12$ \\
\hline $\begin{array}{l}\text { Control for } \\
\text { phentolamine/ } \\
\text { methysergide, } \\
30 \mu \mathrm{g}\end{array}$ & 6 & - & $3.99 \pm 0.09$ & $4.51 \pm 0.32$ & $3.75 \pm 0.10$ & $9.18 \pm 0.82$ & $108 \pm 10$ & $152 \pm 8$ & $321 \pm 21$ & $294 \pm 32$ \\
\hline
\end{tabular}


activated VIP-containing cells (or an unknown cell group in this region) that subsequently communicate with serotonergic and noradrenergic cell groups. Since a nonsignificant attenuation of the pressor response could be obtained by prior 5,7-DHT treatment and the $\mathrm{B} 3$ region supports a pressor response (depressor responses were obtained from the $\mathrm{B} 2$ region), then it is possible that the pressor responses obtained in the present study were mediated in part by the B3 region via activation through cell bodies located in the NRV and in part by an unspecified noradrenergic (adrenergic) cell group. It is less clear that the $\mathrm{Cl}$ cell group contributed to these pressor responses, although it should be noted that the principle adrenergic bundle arising from the C1 group directly traverses regions of microinjection in the present study that support pressor responses. The view that the antinociceptive and cardiovascular effects were not due to spread of the injectate is also indirectly supported by studies examining the spread of lidocaine using the identical microinjection procedure (Sandkühler and Gebhart, 1984; Sandkühler et al., 1987). Based on these studies, we would expect the maximum range of spread to be $0.2-0.9 \mathrm{~mm}$ with these volumes and time parameters (tests $1 \mathrm{~min}$ after the start of the injection), and more likely the spread was between $0.1-0.2 \mathrm{~mm}$. Since the $\mathrm{Cl}$ region is $2-4 \mathrm{~mm}$ from these injection sites, it seems unlikely that the injectate reached this region.

The present experiments also suggest that descending noradrenergic (adrenergic) and serotonergic systems are engaged by neurons projecting from these depressor and pressor regions to produce antinociception. Specifically, combined intrathecal administration of noradrenergic and serotonergic receptor antagonists localized to the level of the lumbar spinal cord was required to eliminate the antinociception resulting from glutamate microinjected into either depressor or pressor areas of the dorsal mcdulla. A similar effect could not be achieved with intrathecal administration of naloxone alone, methysergide alone, or phentolamine alone. With respect to the doses of phentolamine and methysergide required, the present observations are supported by results from a recent study in which the modulatory influence of electrical stimulation of the cervical vagus nerve on the nociceptive tail-flick reflex was examined (Ren et al., 1988). Inhibition of the tail-flick reflex by electrical stimulation of the vagus was affected by the combined intrathecal administration of $30 \mu \mathrm{g}$ each of methysergide and phentolamine. While the dosages required appear great, previous work has established that similar dosages of phentolamine and methysergide are required to antagonize fully the maximum antinociceptive effects produced by the intrathecal administration of their respective agonists (Yaksh and Wilson, 1979; Reddy and Yaksh, 1980; Reddy et al., 1980; Schmauss et al., 1983). The antagonism at these dosages is considered selective inasmuch as there is no apparent antagonism of the effects of the other receptor agonists, e.g., intrathecal administration of adrenergic receptor antagonists do not affect antinociception produced by intrathecal administration of serotonin. Thus, it would seem that the microinjection of glutamate into either the NTS or NRV powerfully activates descending systems of inhibition that require maximally effective dosages of receptor antagonists to attenuate descending modulation. Although the magnitude of the pressor response evoked by glutamate was reduced in the group treated with the combined administration of the greatest doses of phentolamine and methysergide, Experiment 4 showed that the pressor effect is unnecessary to obtain antinociception. Indeed, these data from intrathecal administration of receptor antagonists indicatc that the sympathetic outflow mediating the pressor response evoked by glutamate microinjected into the NRV is partly mediated by the combined release of NE and 5 -HT in the spinal cord.

These data have several implications for our understanding of the role of the NTS in the production of antinociception. The data would account for a previous report (Lohof et al., 1987) that intrathecal administration of methysergide alone failed to affect inhibition of the tail-flick reflex produced by electrical stimulation in the NTS and suggest that such inhibition might have been antagonized by combined administration of noradrenergic and serotonergic receptor antagonists. The present data implicating descending control by noradrenergic and serotonergic substrates are also consistent with sites in the medulla that have been shown to mediate inhibition of the tail-flick reflex produced by electrical stimulation of the vagus. Specifically, the LRN, NRO, NTS, and NRM-MRF all participate in vagally mediated inhibition of the tail-flick reflex, and some of these sites also utilize both noradrenergic and serotonergic descending inhibitory control systems (Sandkühler and Gebhart, 1984; Janss and Gebhart, 1987; Randich and Aicher, 1988; Ren et al., 1988).

However, in the present study we failed to find an effect of intrathecal naloxone administration on the inhibition produced by microinjections of glutamate in the NTS, and inhibition of the tail-flick reflex resulting from electrical stimulation of either the right cervical vagus or the NTS can be attenuated by intrathecal administration of naloxone alone, as indicated previously, or by the combined administration of $30 \mu \mathrm{g}$ each of phentolamine and methysergide (Lohof et al., 1987; Ren et al., 1988). Admittedly, the present studies failed to assess combinations of naloxone with either phentolamine or methysergide, but the failure to obtain an effect with naloxone alone in the present study could be attributed to several possible factors. Microinjections of glutamate may not have affected all of the areas activated by electrical stimulation of either the vagus or the NTS. On the basis of anatomical mapping studies of cardiopulmonary vagal afferents, terminal afferent regions that glutamate would not have directly influenced are area postrema and perhaps some far caudal termination sites (e.g., nucleus retroambiguous and cervical spinal cord). Similarly, it may be the case that the opioid receptor-mediated effect reported previously with electrical stimulation of the vagus stems from activation of subdiaphragmatic afferents that terminate in portions of the medulla not accessed by our microinjection procedure. Conversely, in addition to activating cells of a vagal-inhibitory system, microinjections of glutamate may have also recruited cells of another system utilizing different spinal neurotransmitters, e.g., a carotid sinus-inhibitory system, thereby masking the ability to detect an effect of naloxone alone. With regard to naloxone antagonism of the inhibitory effect of electrical stimulation in the NTS, however, it must always be considered that stimulation of fibers of passage in addition to cell bodies contributed to the opioid receptor involvement previously reported. The precise efficacy of intrathecally administered naloxone in blocking inhibition of the tail-flick reflex produced by electrical stimulation in the NTS has not yet been systematically reported (see Lohof et al., 1987).

The present experiments also failed to find any consistent facilitation of the tail-flick reflex following microinjections of glutamate into the NTS. Ren et al. (1988) reported that facilitation of the tail-flick reflex was obtained during electrical stimulation of the cervical vagus when intensities of stimulation were 
$0.2-0.6$ of the threshold currents required for inhibition of the tail-flick reflex. It is our view that facilitation of the tail-flick reflex may well be observed with microinjections of glutamate in the NTS when shorter test intervals intervene between the microinjection of glutamate and the tail-flick test trial, i.e., $<1$ min. Specifically, we speculate that the facilitatory effect not only depends upon activation of vagal afferents different from those responsible for inhibition, but also is shorter-lived than the inhibitory effect, and hence, not readily detected with the present procedures.

Experiment 4 ruled out the possibility that elevations in arterial blood pressure alone contributed to the antinociception produced by microinjection of glutamate into the NRV and showed that such elevations reflect increased sympathetic outflow. This study also ruled out the possibility that the significant reduction in the pressor response in the combined intrathecal antagonist condition of Experiment 3 was responsible for the loss of the antinociception. Instead, it indicates that the increased sympathetic outflow, like the antinociception, is a consequence of engaging descending noradrenergic and serotonergic systems. Finally, Experiment 4 also indirectly indicates that the antinociception accompanying the depressor responses evoked by glutamate microinjected into the NTS is not a consequence of the peripheral decrease in arterial blood pressure. Hexamethonium administration decreased arterial blood pressure to levels lower than those produced with microinjections of glutamate, but the tail-flick reflex was unaffected.

In conclusion, the present findings provide evidence that the NTS and the NRV may play a role in pain modulation and serve to integrate visceral-visceral, visceral-somatic, and somatic-visceral responses.

\section{References}

Aimone, L. D., and G. F. Gebhart (1986) Stimulation-produced spinal inhibition from the midbrain in the rat is mediated by an excitatory amino acid neurotransmitter in the medial medulla. J. Neurosci. 6: 1803-1813.

Ammons, W. S., R. W. Blair, and R. E. Foreman (1983a) Vagal afferent inhibition of primate thoracic spinothalamic neurons. J. Neurophysiol. 50: 926-940.

Ammons, W. S., R. W. Blair, R. D. Foreman (1983b) Vagal afferent inhibition of spinothalamic cell responses to sympathetic afferents and bradykinin in the monkey. Circ. Res. 53: 603-612.

Du, H. J., and S. Y. Zhou (1987) Descending modulation of nociceptive transmission by solitary nucleus in cat spinal cord neurons. Pain (Suppl.) 4: S32.

Goodchild, A. K., R. A. L. Dampney, and R. Bandler (1982) A method for evoking physiological responses by stimulation of cell bodies, but not axons of passage, within localized regions of the central nervous system. J. Neurosci. Methods 6: 351-363.

Janss, A. J., and G. F. Gebhart (1987) Spinal monoaminergic receptors mediate the antinociception produced by glutamate in the medullary lateral reticular nucleus. J. Neurosci. 7: 2862-2873.

Johnson, J. L. (1972) Glutamic acid as a synaptic neurotransmitter in the nervous system. A review. Brain Res. 37: 1-19.
Kalia, M., and J. M. Sullivan (1982) Brainstem projections of sensory and motor components of the vagus nerve in the rat. J. Comp. Neurol. 211: $248-264$

Leibstein, A. G., R. Dermietzel, I. M. Willenberg, and R. Pauschert (1985) Mapping of different neuropeptides in the lower brainstem of the rat with special reference to the ventral surface. J. Auton. Nerv. System 14: 299-213.

Lewis, J. W., G. Baldrighi, and H. Akil (1987) A possible interface between autonomic function and pain control: Opioid analgesia and the nucleus tractus solitarius. Brain Res. 424: 65-70.

Lohof, A. M., M. M. Morgan, J.-H. Sohn, and J. C. Liebeskind (1987) Stimulation produced analgesia from the nucleus tractus solitarius in the rat: Spinal pharmacology. Pain (Suppl.) 4: S287.

Maixner, W., and A. Randich (1984) Role of the right vagal nerve trunk in antinociception. Brain Res. 298: 374-377.

Menetrey, D., and A. I. Basbaum (1987) Spinal and trigeminal projections to the nucleus of the solitary tract: A possible substrate for somatovisceral and viscerovisceral reflex activation. J. Comp. Neurol. 255: 439-450.

Minson, J. B., J. P. Chalmers, A. C. Caon, and B. Renaud (1987) Separate areas of rat medulla oblongata with populations of serotoninand adrenaline-containing neurons alter blood pressure after L-glutamate stimulation. J. Auton. Nerv. System 19: 39-50.

Newman, D. B. (1985) Distinguishing rat brainstem reticulospinal nuclei by their neuronal morphology. I. Medullary nuclei. J. Hirnforsch. 2: 187-226.

Paxinos, G., and C. Watson (1986) The Rat Brain in Stereotaxic Coordinates, Academic, New York.

Randich, A., and S. A. Aicher (1988) Medullary substrates mediating antinociception produced by electrical stimulation of the vagus. Brain Res. 445: 68-76.

Randich, A., and W. Maixner (1984a) Interactions between cardiovascular and pain regulatory systems. Neurosci. Biobehav. Rev. 8: 343-367.

Randich, A., and W. Maixner (1984b) [D-Ala $\left.{ }^{2}\right]$-methionine enkephalinamide reflexively induces antinociception by activating vagal afferents. Pharmacol. Biochem. Behav. 21 441-448.

Reddy, S. V. R., and T. L. Yaksh (1980) Spinal noradrenergic terminal sytem mediated antinociception. Brain Res. 289: 391-402.

Reddy, S. V. R., J. L. Maderdrut, and T. L. Yaksh (1980) Spinal cord pharmacology of adrenergic agonist-mediated antinociception. J. Pharmacol. Exp. Ther. 213: 525-533.

Ren, K., A. Randich, and G. F. Gebhart (1988) Vagal afferent modulation of a nociceptive reflex in rats: Involvement of spinal opioid and monoamine receptors. Brain Res. 446: 285-294.

Sandkühler, J., and G. F. Gebhart (1984) Relative contributions of the nucleus raphe magnus and adjacent medullary reticular formation to the inhibition of stimulation in the periaqueductal gray of a spinal nociceptive reflex in the pentobarbital-anesthetized rat. Brain Res. 305: 77-87

Sandkühler, J., B. Maisch, and M. Zimmerman (1987) The use of local anaesthetic microinjections to identify central pathways: A quantitative evaluation of the time course and extent of the neuronal block. Exp. Brain Res. 68: 168-178.

Schmauss, C., D. L. Hammond, J. W. Ochi, and T. L. Yaksh (1983) Pharmacological antagonism of the antinociceptive effects of serotonin in the rat spinal cord. Eur. J. Pharmacol. 90: 349-357.

Yaksh, T. L., and T. A. Rudy (1976) Chronic catheterization of the spinal subarachnoid space. Physiol. Behav. 17: 1031-1036.

Yaksh, T. L., and P. R. Wilson (1979) Spinal serotonin terminal system mediates antinociception. J. Pharmacol. Exp. Ther. 208: 446-453. 